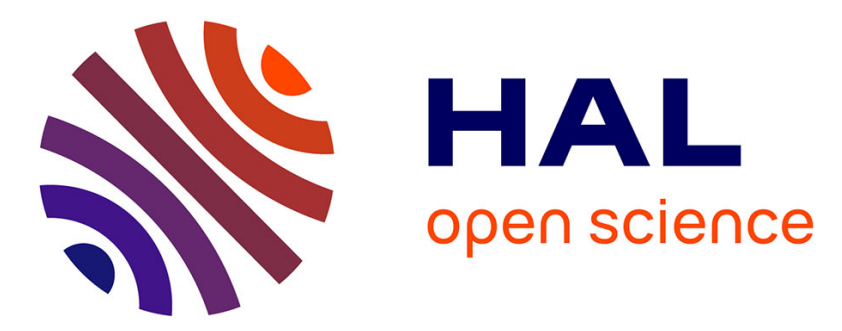

\title{
Ultracompact Binary Permanent Rare-Earth Magnet with 1.25-T Center Field and Fast-Decaying Stray Field
}

\author{
Jean-Marie Poumirol, Adrien Bercher, Tetiana Slipchenko, Ivan \\ Maggio-Aprile, Christoph Renner, Alexey B Kuzmenko
}

\section{- To cite this version:}

Jean-Marie Poumirol, Adrien Bercher, Tetiana Slipchenko, Ivan Maggio-Aprile, Christoph Renner, et al.. Ultracompact Binary Permanent Rare-Earth Magnet with 1.25-T Center Field and Fast-Decaying Stray Field. Physical Review Applied, 2021, 16, 10.1103/physrevapplied.16.044012 . hal-03455372

\author{
HAL Id: hal-03455372 \\ https://hal.science/hal-03455372
}

Submitted on 29 Nov 2021

HAL is a multi-disciplinary open access archive for the deposit and dissemination of scientific research documents, whether they are published or not. The documents may come from teaching and research institutions in France or abroad, or from public or private research centers.
L'archive ouverte pluridisciplinaire HAL, est destinée au dépôt et à la diffusion de documents scientifiques de niveau recherche, publiés ou non, émanant des établissements d'enseignement et de recherche français ou étrangers, des laboratoires publics ou privés. 


\title{
Ultracompact Binary Permanent Rare-Earth Magnet with 1.25-T Center Field and Fast-Decaying Stray Field
}

\author{
Jean-Marie Poumirolø,${ }^{1, \dagger}$ Adrien Bercher, ${ }^{1}$ Tetiana Slipchenko, ${ }^{2,3}$ Ivan Maggio-Aprile, ${ }^{1}$ \\ Christoph Renner $\odot,{ }^{1}$ and Alexey B. Kuzmenko ${ }^{1,{ }^{*}}$ \\ ${ }^{1}$ Department of Quantum Matter Physics, University of Geneva, Geneva 1211, Switzerland \\ ${ }^{2}$ Instituto de Nanociencia y Materiales de Aragón (INMA) CSIC-Universidad de Zaragoza, Zaragoza 50009, Spain \\ ${ }^{3}$ Departamento de Física de la Materia Condensada, Universidad de Zaragoza, Zaragoza 50009, Spain
}

(Received 20 July 2021; revised 25 August 2021; accepted 22 September 2021; published 11 October 2021)

\begin{abstract}
We present a very-compact bicomponent-permanent-magnet design capable of generating $1.25 \mathrm{~T}$ in a small volume, significantly above the $0.6 \mathrm{~T}$ available from a single uniformly magnetized permanent magnet. In addition to the enhanced maximum field, our design drastically limits the stray field present around a standard permanent magnet. These features make it suitable for retrofitting existing experiments with a substantial magnetic field, in particular, scanning probes and optical, Raman, and photoemission spectroscopy, in diverse environments, from ambient to ultrahigh vacuum and over a wide temperature range.
\end{abstract}

DOI: 10.1103/PhysRevApplied.16.044012

\section{INTRODUCTION}

Generating strong magnetic fields (1 $\mathrm{T}$ and above) typically involves cumbersome and heavy electrical magnets or cryogenic superconducting coils [1-5]. Such equipment is not compatible with all experiments and does not allow magnetic fields to be easily retrofitted to existing experiments. Permanent magnets offer an attractive alternative for a number of experiments, albeit being nontunable.

The highest magnetic fields that can be generated by a single-piece rare-earth magnet are limited to about $0.6 \mathrm{~T}$. This limit can be overcome by a clever assembly of individual magnets magnetized in different directions. Examples of such combined devices are the Halbach arrays [6-8] widely used in particle accelerators and levitating-train technology, as well as magnetic modules for unlocking antitheft tags in supermarkets [9]. Following this strategy, we propose a very-compact permanent magnet only 5.5 $\mathrm{mm}$ in diameter and $3.67 \mathrm{~mm}$ high, consisting of two oppositely magnetized elements generating a magnetic field of about $1.25 \mathrm{~T}$.

The paper is organized as follows. In Sec. II, we introduce the basic physical principle behind the design. In Sec. III, we describe the prototype device. In Sec. IV, we apply it to produce the Abrikosov vortex lattice in $\mathrm{NbSe}_{2}$ and visualize it using STM. In Sec. V, we present numerical simulations of the spatial distribution of the

\footnotetext{
*Alexey.Kuzmenko@unige.ch

$\dagger$ Present address: CEMES, University of Toulouse, CNRS, Toulouse 31000 , France.
}

magnetic field. Finally, in Sec. VI, we discuss the advantages and drawbacks of the device and outline possible future developments.

\section{THEORETICAL PRINCIPLE}

We start by considering a textbook case of point magnetic dipole $\vec{m}[10,11]$. We suppose that it is located at a point $\vec{r}=(x, y, z)$ and oriented along the $z$ axis, such as $\vec{m}=m \vec{z}$. At another point $\vec{r}^{\prime}=\left(x^{\prime}, y^{\prime}, z^{\prime}\right)$, it generates the magnetic field given by

$$
\vec{B}\left(\vec{r}, \overrightarrow{r^{\prime}}\right)=\frac{\mu_{0} m}{4 \pi\left|\overrightarrow{r^{\prime}}-\vec{r}\right|^{3}}[3 \vec{n}(\vec{z} \vec{n})-\vec{z}],
$$

where $\mu_{0}=4 \pi \times 10^{7} \mathrm{H} / \mathrm{m}$ is the permeability of vacuum and $\vec{n}=\left(\overrightarrow{r^{\prime}}-\vec{r}\right) /\left|\overrightarrow{r^{\prime}}-\vec{r}\right|$ is the unit vector pointing from $\vec{r}$ to $\vec{r}^{\prime}$. In particular, the $z$ component of the field at $\vec{r}^{\prime}$ equals

$$
B_{z}\left(\vec{r}, \overrightarrow{r^{\prime}}\right)=\frac{\mu_{0} m}{4 \pi\left|\overrightarrow{r^{\prime}}-\vec{r}\right|^{3}}\left[3(\vec{z} \vec{n})^{2}-1\right] .
$$

One can see that $B_{z}$ is positive within the "optimal cone" defined by the inequality $(\vec{z} \vec{n})^{2}>1 / 3$ and negative in the outside space [Fig. 1(a)]. One half of the opening angle of the cone is $\alpha_{\text {opt }}=\arccos (1 / \sqrt{3}) \approx 54.7^{\circ}$.

We are interested in maximizing $B_{z}$ at the origin $\mathrm{O}$ $\left(x^{\prime}=y^{\prime}=z^{\prime}=0\right)$ created by a permanent magnet of a finite size, which can be mathematically represented as a superposition of a continuum of point magnets with the volume magnetization $\vec{M}=d \vec{m} / d V$. From the above statements it follows that if the permanent magnet occupies 
(a)

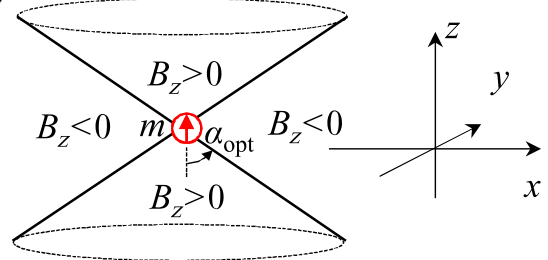

(b)

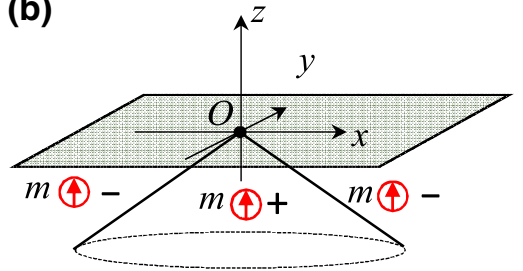

(c)

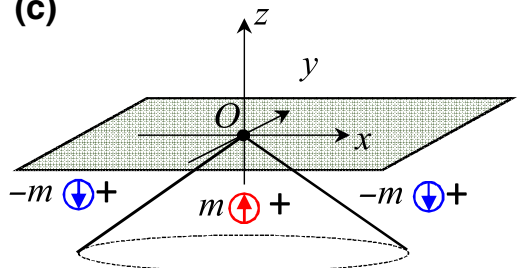

FIG. 1. Basic idea behind the magnetic device. (a) Optimal cone separating the spatial regions with positive and negative values of the vertical component of the magnetic field generated by a vertically aligned point magnetic dipole in the cone center. (b) When the lower half of the space $(z<0)$ is filled with magnetic dipoles along the same direction, this will generate a low magnetic field at the origin, as contributions from the region inside and outside the optimal cone will contribute with opposite signs, as indicated by \pm signs next to the dipole. (c) When the spatial regions inside and outside the optimal cone are filled with magnetic dipoles aligned in opposite directions, the magnetic field at the origin will be maximized.

the half-space $z<0$ and is magnetized uniformly along the $z$-axis, the contributions from the magnetic moments inside and outside the optimal cone tend to compensate each other [Fig. 1(b)] (in fact, they exactly cancel each other for an infinitely wide slab). Alternatively, if the magnetic moments inside and outside the cone boundary are parallel but oriented in the opposite directions then they all contribute to $B_{z}(0,0,0)$ with the same direction, therefore increasing the field at this point [Fig. 1(c)].

The first idea following from the above consideration is to create a high-axial magnetic field in the vicinity of a given point (typically on the surface of the studied sample) by oppositely orienting the magnetic moments inside and outside the optimal cone with an apex close to this point. Technically, this can be done by using two oppositely magnetized pieces with geometrically complementary shapes as close as possible to the optimal cone.

The magnetic field generated near the apex of an ideal optimal cone is highly nonuniform, making it impractical for microscopy. The second idea behind the design of the present device is to use a truncated cone (frustum), while keeping the cone angle close to $\alpha_{\text {opt }}$. Truncating the cone has the advantage that the axial magnetic field is still quite high but relatively more homogeneous in the region around the truncated area.

\section{BICOMPONENT HIGH-FIELD PERMANENT-MAGNET DESIGN}

Taking into account the above considerations, we fabricate a device made of two Nd-Fe-B magnet pieces, which are shown in Fig. 2. Figures 2(a) and 2(b) present the actual dimensions of the inner truncated cone and the corresponding outer part, respectively (which tightly fit to each other); a photograph of the assembled device is given in Fig. 2(c). The external diameter and height of the device are $D=5.5$ $\mathrm{mm}$ and $h=3.67 \mathrm{~mm}$, respectively; the truncation diameter is $d=0.3 \mathrm{~mm}$. For these specific dimensions (chosen based on the ease of production), the cone angle is about $35^{\circ}$, which is smaller than the optimal value of $54.7^{\circ}$. (a)
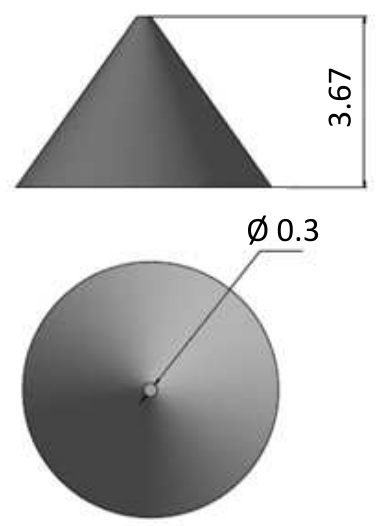

(b)

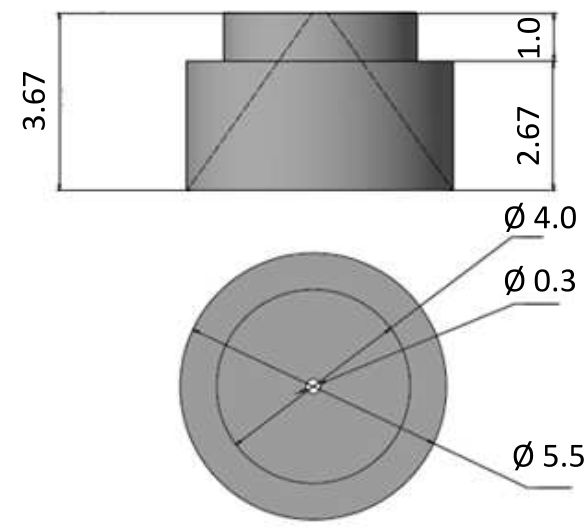

(c)

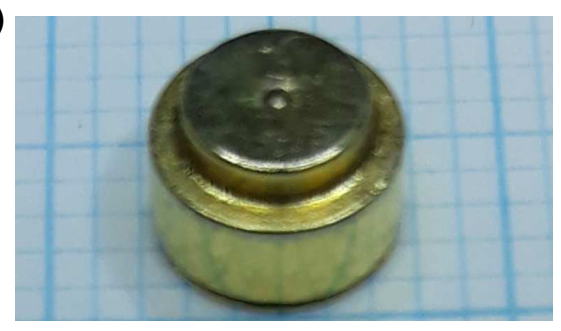

(d)

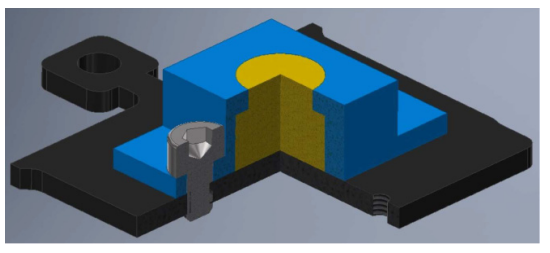

FIG. 2. Prototype magnet device. (a),(b) Dimensions (in mm) of inner and outer parts, respectively. (c) Photograph of the assembled device on millimeter paper. (d) Computer-aided design drawing of the magnet mounting on the STM sample holder. Magnet, fixation piece, and STM holder are shown in yellow, blue, and black respectively. 
Therefore, the generated field is somewhat weaker that what would be achieved with the fully optimized geometry (as discussed in Sec. II). The 1-mm-deep circular groove around the outer piece is made to facilitate mechanical fixation of the device on a sample holder. As the total weight of this device is only $640 \mathrm{mg}$, it can be put directly onto any piezo scanner without compromising its dynamic characteristics.

Two different grades of Nd-Fe-B are chosen for the inner and outer pieces. The inner piece is made of Nd-Fe-B$N 55$ (remanent magnetization $M=1.49 \mathrm{~T}$, coercive field $H_{c, j}=11000 \mathrm{Oe}$ ), which has one of the largest values of $M$. On the other hand, grade $N 48$ is used for the outer piece ( $\left.M=1.40 \mathrm{~T}, H_{c, j}=12000 \mathrm{Oe}\right)$ with a larger coercive field to reduce the risk of demagnetization of this part of the magnet near the cone apex.

\section{MEASURING THE MAGNETIC FIELD USING STM}

Measuring the magnetic field of our device with an expected homogeneity area in the order of $100 \mu \mathrm{m}$ is not straightforward. For example, it is not possible to do it with conventional millimeter-sized Hall sensors. Among the techniques offering better spatial resolution and the ability to measure the absolute value are scanning superconducting quantum interference device microscopy [12-14] and nitrogen-vacancy-center sensing [15-18]. However, none of these options are suitable for us, either due to availability of the instrument or because of the limits on measurable magnetic fields.

To quantify the magnetic field very locally and precisely, we use instead a scanning tunneling microscope (STM) to visualize the Abrikosov flux vortex lattice on a $\mathrm{NbSe}_{2}$ single crystal in the superconducting state $\left(T_{c}=7.2 \mathrm{~K}\right)$ [19]. The exceptional precision of this technique is based on the fact that each vortex holds exactly one flux quantum $\Phi_{0}=h / 2 e=2.067 \times 10^{-15} \mathrm{~T} \mathrm{~m}^{2}[20,21]$. Therefore, one can determine the magnetic field by simply measuring the density of vortices.

In our experiment, the magnet device is fixed to the STM sample-holder plate. A thin crystal of $\mathrm{NbSe}_{2}$ (approximately $0.1 \mathrm{~mm}$ ) is glued directly on top of the magnet with silver paint and cleaved under an ambient atmosphere. Afterwards, it is immediately introduced into the STM space and evacuated to a pressure better than $10^{-7}$ mbar. The STM measurements are done in a ${ }^{3} \mathrm{He}$ cryostat [22] at a base temperature of $400 \mathrm{mK}$ in a cryogenic vacuum of $<10^{-8}$ mbar.

The detection of individual flux vortices with the STM relies on the fact that the quasiparticle excitation spectrum exhibits nonsuperconducting characteristics close to the vortex center. The excitation spectrum is measured via differential conductance spectroscopy, $\sigma(V)=d I(V) / d V$, where $I(V)$ is the tunneling current recorded with an
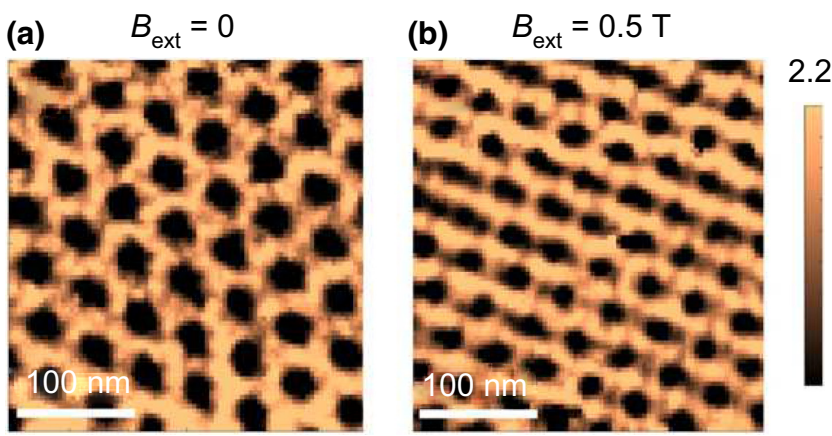

FIG. 3. $300 \times 300 \mathrm{~nm}^{2}$ STM images of flux vortices on $\mathrm{NbSe}_{2}$ at $T=0.4 \mathrm{~K}$. Color scale corresponds to the conductance ratio $R=\sigma\left(V_{g}\right) / \sigma(0)$. (a) Vortex lattice generated by the field of the permanent magnet. (b) Vortex lattice in the same region after applying an additional magnetic field, $B_{\text {ext }}$, of $0.5 \mathrm{~T}$ using the superconducting coil of the cryogenic setup. Slight misalignment of the lattice relative to the one in (a) is possibly due to vortex creep occurring throughout the measurement.

interrupted feedback loop, and $V$ the tip-sample bias voltage. Since the most significant changes in the conductance spectra acquired inside vortex cores occur at $V=0$ and $V_{g}=\Delta / e$, where $\Delta \sim 1.5 \mathrm{meV}$ is the superconducting gap, the optimal STM contrast is obtained by mapping the ratio $R=\sigma\left(V_{g}\right) / \sigma(0)$ [23].

Figure 3(a) shows the STM $R$ map on a $300 \times 300 \mathrm{~nm}^{2}$ area. We can estimate the vertical magnetic field, $B$, using the flux-quantization relation $B=\Phi_{0} n / S$, where $n / S$ is the density of vortices per unit of area. The total number of flux vortices in Fig. 3(a) is $55 \pm 2$, leading to $B=(1.26 \pm 0.05) \mathrm{T}$. Alternatively, we can estimate the average distance between the vortices $l=(44 \pm 2) \mathrm{nm}$ and use the formula $B=2 \times 3^{-1 / 2} \Phi_{0} / l^{2}$ valid for the triangular lattice. This gives a very close result of $B=(1.24 \pm$ $0.05) \mathrm{T}$. Thus, we conclude that the field of our device is $B=1.25 \pm 0.05 \mathrm{~T}$.

To see whether the device can sustain an external field, we additionally apply $B_{\text {ext }}=0.5 \mathrm{~T}$ using the superconducting coil of our cryogenic setup. The STM map obtained under this condition is shown in Fig. 3(b). Using the same method, we find that the total field is now $B \approx$ $(1.8 \pm 0.05) \mathrm{T}$. The fact that the field intensity has indeed increased by the amount given by $B_{\text {ext }}$ indicates that the external field adds linearly to the intrinsic field and does not have a demagnetization effect on the device. This demonstrates that the magnet can be used in combination with a source of external field, to further raise the field intensity and achieve additional tunability.

\section{NUMERICAL SIMULATIONS}

We now proceed with numerical simulations of the generated magnetic field around our magnet device. 
According to Eq. (1), the total field generated by a permanent magnet with uniform remanent magnetization $M$, occupying a three-dimensional region $V$, can be calculated using the following three-dimensional integration:

$$
\vec{B}\left(\overrightarrow{r^{\prime}}\right)=\frac{\mu_{0}}{4 \pi} \int_{V} \frac{M(\vec{r})}{\left|\overrightarrow{r^{\prime}}-\vec{r}\right|^{3}}[3 \vec{n}(\vec{z} \vec{n})-\vec{z}] d^{3} \vec{r},
$$

Due to the axial symmetry of the device, the field depends only on the radial distance $r^{\prime}=\left(x^{\prime 2}+y^{\prime 2}\right)^{1 / 2}$ and height $z^{\prime}$. For the same reason, only the radial and axial components of the field, $B_{r}$ and $B_{z}$, need to be calculated. Equation (3), in this case, can be reduced to a two-dimensional integral by using a cylindrical coordinate system and performing the integration over the azimuth angle analytically. As a result, the magnetic field can be calculated by a numerical integration over the half-cross-section $S / 2$ of the device cut by a plane, which contains the symmetry axis

$$
B_{r, z}\left(r^{\prime}, z^{\prime}\right)=\frac{\mu_{0} M}{4 \pi r^{\prime 2}} \iint_{S / 2} Q_{r, z}\left(\frac{r^{\prime}}{r}, \frac{z^{\prime}-z}{r}\right) d r d z,
$$

where

$$
\begin{aligned}
& Q_{r}(\xi, \zeta)=\frac{2 \zeta \xi}{(\rho+2 \xi)^{3 / 2}(\rho-2 \xi)}\left\{\frac{8 \rho \xi^{2}-\rho^{2}-12 \xi^{2}}{\rho-2 \xi} E\left(\frac{4 \xi}{\rho+2 \xi}\right)+\left(\rho-2 \xi^{2}\right) K\left(\frac{4 \xi}{\rho+2 \xi}\right)\right\}, \\
& Q_{z}(\xi, \zeta)=\frac{4 \xi^{2}}{(\rho+2 \xi)^{3 / 2}(\rho-2 \xi)}\left\{\frac{4 \xi^{2}-\rho^{2}+4 \zeta^{2} \rho}{\rho-2 \xi} E\left(\frac{4 \xi}{\rho+2 \xi}\right)-\zeta^{2} K\left(\frac{4 \xi}{\rho+2 \xi}\right)\right\},
\end{aligned}
$$

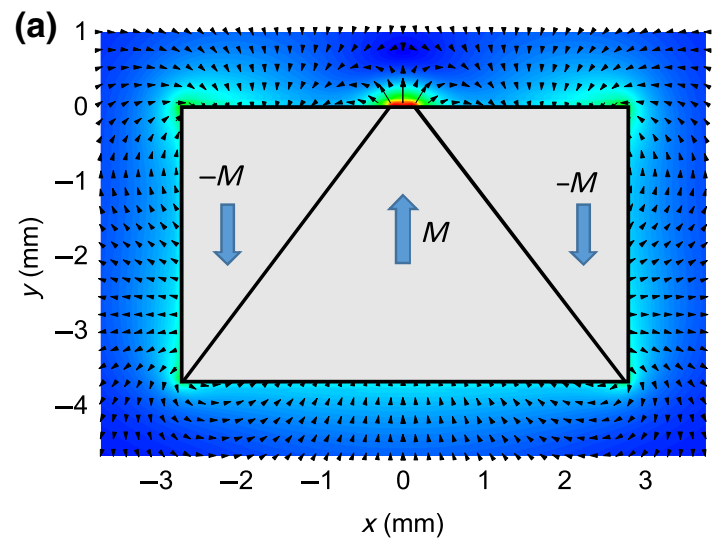

(c)

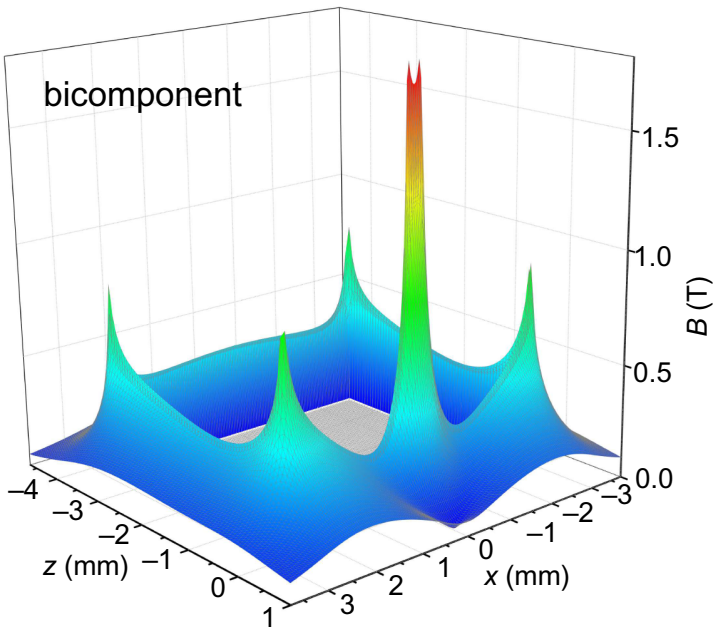

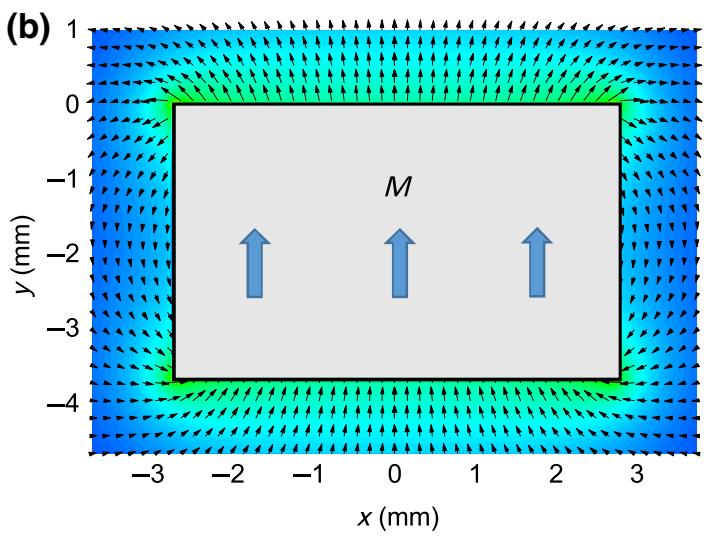

(d)

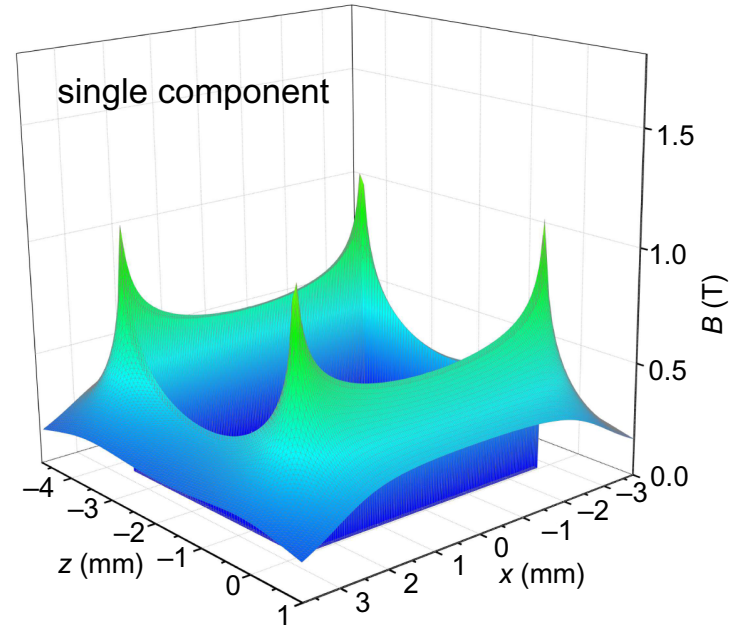

FIG. 4. Simulated magnetic field distribution around the magnet. Magnetic field distribution around (a) bicomponent device and (b) single-component magnet of the same size ( $y=0$ cross section is shown). Color denotes the absolute field intensity while arrows show the field direction. (c),(d) Three-dimensional (3D) surface plot of absolute field intensity from panels (a),(b), respectively, with the same color scale. Field inside the magnet (gray area) is not calculated. 
and $\rho=1+\xi^{2}+\zeta^{2}$. Here, $K(m)$ and $E(m)$ are the complete elliptic integrals of the first and second kind. For the points on the symmetry axis, these expressions reduce to

$$
Q_{r}(\zeta, \xi=0)=0, \quad Q_{z}(\zeta, \xi=0)=\pi \frac{2 \zeta^{2}-1}{\left(1+\zeta^{2}\right)^{5 / 2}}
$$

Using these equations, we calculate the magnetic field for a bicomponent device with the same dimensions as in Fig. 2 and compare it with the result for a single-component magnet of the same dimensions. For the sake of simplicity, we set the remanent magnetization, $M$, of both pieces to the same value $(1.5 \mathrm{~T})$, even though the magnetic grades used are slightly different, as mentioned above. We also ignore the external groove, as it has only a weak effect on the field distribution near the maximum. Figure 4(a) shows the $x-z$ cross section of the device and the maps of the intensity (color) and direction (arrows) of the outside field. Additionally, Fig. 4(c) presents the absolute intensity as a 3D plot. Figures 4(b) and 4(d) refer to the same calculation for a single-component magnet of the same dimensions. One can see that, in contrast to the single magnet, (i) the magnetic field of our device manifests a sharp peak, reaching almost $2 \mathrm{~T}$ near the field center $(x=z=0)$, and (ii) the field decreases quickly with the distance from the surface.

In Fig. 5, we present the field of our device and a single magnet in the area of $1 \mathrm{~mm}$ around the field center. Figure 5(a) shows the $z$ dependence of $B_{z}$ on the magnet axis (where the radial component is zero). Remarkably, at $z=0$, the field of the bicomponent magnet $(1.9 \mathrm{~T})$ is about 3 times higher than in the single magnet $(0.6 \mathrm{~T})$. The second difference is that the field decays in height much faster in the bicomponent device (in fact, it even crosses zero).
The field decreases by a factor of 2 already $0.15 \mathrm{~mm}$ above the magnet and falls below $0.1 \mathrm{~T}$ above $0.5 \mathrm{~mm}$. The field of the single magnet on the same scale reduces only by $10 \%$.

As mentioned, a somewhat suboptimal value of the cone angle is used in the present device $\left(35^{\circ}\right.$ instead of $\left.54.7^{\circ}\right)$. The inset of Fig. 5(a) shows the calculated angle dependence of the vertical component of the magnetic field at $x=0$ and $z=0.1 \mathrm{~mm}$. One can see that the field for the optimal angle should be about $25 \%$ higher.

In Fig. 5(b), we demonstrate the $x$ dependence of $B_{z}$ at different heights, $z$, above the magnet. One can see that the field of the bicomponent magnet is relatively homogeneous within $0.1 \mathrm{~mm}$ around the center (which is much larger than the image dimensions in typical local probe experiments) but then decays rather quickly in a fashion similar to that in Fig. 5(a). We notice that on the shown scale of $1 \mathrm{~mm}$ the single magnet generates an almost uniform field. Finally, in Fig. 5(c), we present the $x$ dependence of the radial field, $B_{r}$. It is zero on the symmetry axis $(x=0)$, shows a rather linear growth up to $x=0.1 \mathrm{~mm}$, and reaches a maximum at a larger value of $x$ (depending on $z$ ). The radial field of the single-component magnet increases significantly slower.

\section{DISCUSSION}

The most important result of our work is a demonstration that magnetic fields well above $1 \mathrm{~T}$ are achievable with a compact and light permanent magnet that can be mounted essentially on any scanning probe or optical microscope. This makes a viable alternative to placing the entire microscopy setup inside a big magnet. The field
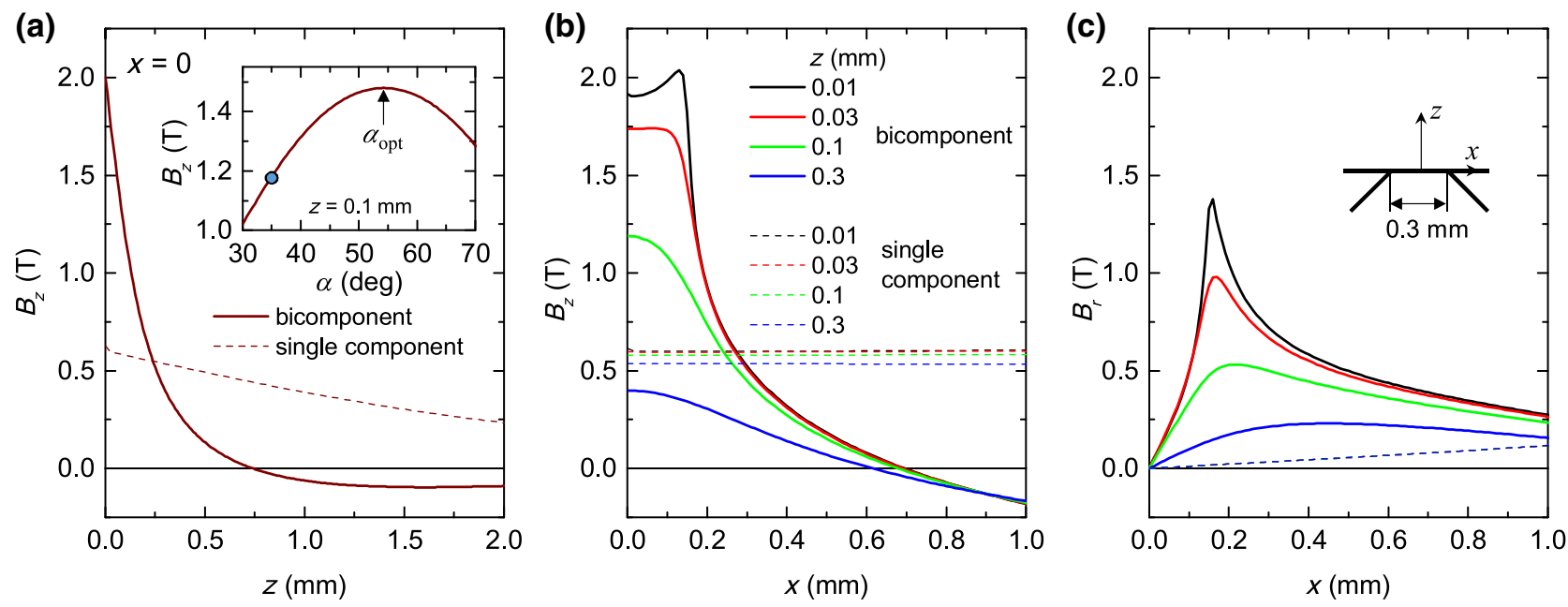

FIG. 5. Calculated magnetic field in the vicinity of the field maximum. (a) $B_{z}$ as a function of $z$ on the magnet's axis ( $x=0$ ). Solid lines correspond to the bicomponent magnet with the same dimensions as in Fig. 2, and dashed curves are obtained for a singlecomponent magnet of the same size. Inset shows the dependence of $B_{z}$ on the cone angle for $z=0.1 \mathrm{~mm}$, while the circle denotes the actual angle in our device $\left(35^{\circ}\right)$. (b) $B_{z}$ and (c) $B_{r}$ as a function of $x$ for different values of $z(0.01,0.03,0.1$, and $0.3 \mathrm{~mm})$. 
intensity measured using the STM (Sec. IV) agrees reasonably well with simulations (Sec. V). Indeed, from Fig. 5, we can see that $B_{z} \approx 1.25 \mathrm{~T}$ deduced from the density of the flux vortices should be observed $0.07 \mathrm{~mm}$ above the magnet, which matches the estimated thickness of our sample. This agreement tells us that the assumptions made in the simulation, such as uniform magnetization along the symmetry axis and the absence of demagnetization near the hot spot, are correct. Notably, according to the same simulations, levels of $B_{z}>1.5 \mathrm{~T}$ should be achievable for $z<0.01 \mathrm{~mm}$.

Employing the two-component design not only increases the maximum field but also reduces field leakage (stray field). Physically, this follows from the fact that the fields created by oppositely magnetized pieces add up at the cone apex but compensate for each other at large distances. If the compensation, which can be achieved by design, is exact, then the field decay will be as fast as that of a quadrupole. The low-leakage field not only improves magnetic compatibility with the components of the existing setups but may also enable alternative applications. For example, it would be interesting to explore the possibility of using such a magnet in angular-resolved photoemission spectroscopy, where the photoemitted electrons quit the magnetic field region quickly enough that their trajectories are not significantly affected by the Lorentz force.

It is worth noting that using a permanent-magnet device has specific advantages in the cryogenic regime. First, it provides a highly stable magnetic field and removes the risk of quenching or current instabilities, which are present in the case of superconducting coils. Second, it enables field-cooled thermal cycling or temperature-dependent measurements under finite magnetic fields over a temperature range not achievable with certain superconductingcoil microscopy setups.

Unavoidably, the present design also has drawbacks. First, the fast decay of the field intensity with the distance from the surface limits the applications of the present device to thin samples. Second, even though the area where the field is uniform is sufficiently large for the image size, there is a need to carefully align the region of interest with respect to the field center. A possible solution is to scale up the device dimensions, which will also proportionally increase the region of the field homogeneity, without changing the maximum field intensity. This follows from the fact that Eq. (4) is invariant with respect to the simultaneous scaling operation: $z \rightarrow \alpha z, r \rightarrow \alpha r$.

A third problem is the lack of in situ field variation, which does not allow, in particular, to set it to zero at a given sample point. However, in some experiments, this is not needed, as other physical parameters can be used for comparative or reference measurements, such as temperature, illumination conditions, electrical current, and gate voltage. Furthermore, under ambient conditions, it should be relatively easy to remove the device or to slide the sample on top of the magnet. Finally, on larger-area samples, the very-limited stray field enables one to select zero-field regions. This feature can actually be used to perform field-dependent measurements by moving the imaging area. In the future, field variation could be achieved by combining the present magnetic device with a modulating electromagnetic coil (normal state or superconducting, depending on the temperature conditions) or by allowing for in situ vertical or horizontal mechanical displacement of the magnet with respect to the sample.

Data that support the findings of this study are available from the corresponding author upon reasonable request.

\section{ACKNOWLEDGMENTS}

We thank Julien Levallois for useful discussions and A. Guipet for technical assistance. This work is financed by the EU Project "Graphene Flagship" (CNECT-ICT604391) and UNIGE/Unitec via the Innogap program.

[1] C. Faugeras, M. Amado, P. Kossacki, M. Orlita, M. Kühne, A. A. L. Nicolet, Yu. I. Latyshev, and M. Potemski, Magneto-Raman Scattering of Graphene on Graphite: Electronic and Phonon Excitations, Phys. Rev. Lett. 107, 036807 (2011).

[2] S. Misra, B. B. Zhou, I. K. Drozdov, J. Seo, L. Urban, A. Gyenis, S. C. J. Kingsley, H. Jones, and A. Yazdani, Design and performance of an ultrahigh vacuum scanning tunneling microscope operating at dilution refrigerator temperatures and high magnetic fields, Rev. Sci. Instrum. 84, 103903 (2013).

[3] W. Tao, S. Singh, L. Rossi, J. W. Gerritsen, B. L. M. Hendriksen, A. A. Khajetoorians, P. C. M. Christianen, J. C. Maan, U. Zeitler, and B. Bryant, A low-temperature scanning tunneling microscope capable of microscopy and spectroscopy in a bitter magnet at up to $34 \mathrm{~T}$, Rev. Sci. Instrum. 88, 093706 (2017).

[4] J. Tao, L. Shen, S. Hosaka, Y. Yin, T. Miura, and W. Hou, Scanning near field circular polarization optical microscope for measuring magnetic nanodot arrays, Sci. Adv. Mater. 9, 151 (2017).

[5] S. Hosaka, H. Sone, Y. Yin, and T. Miura, Patent WO 2016/024629 A1 (2016).

[6] K. Halbach, Design of permanent multipole magnets with oriented rare earth cobalt material, Nuclear Instr. Methods 169, 1 (1980).

[7] K. Halbach, Application of permanent magnets in accelerators and electron storage rings, J. Appl. Phys. 57, 3605 (1985).

[8] J. E. Hilton, S.M. McMurry, An adjustable linear halbach array, J. Magn. Magn. Mater. 324, 2051 (2012).

[9] S. Zhang, Patent DE 102007002065 A1 (2007).

[10] J. D. Jackson, Classical Electrodynamics (John Wiley \& Sons, New York, 1998), 3rd ed.

[11] L. D. Landau and E. M. Lifshitz, The Classical Theory of Fields (Pergamon Press, Oxford, 1971). 
[12] R. C. Black, A. Mathai, F. C. Wellstood, E. Dantsker, A. H. Miklich, D. T. Nemeth, J. J. Kingston, and J. Clarke, Magnetic microscopy using a liquid nitrogen cooled $\mathrm{YBa} 2 \mathrm{Cu} 3 \mathrm{O} 7$ superconducting quantum interference device, Appl. Phys. Lett. 62, 2128 (1993).

[13] C. C. Tsuei, J. R. Kirtley, C. C. Chi, Lock See Yu-Jahnes, A. Gupta, T. Shaw, J. Z. Sun, and M. B. Ketchen, Pairing Symmetry and Flux Quantization in a Tricrystal Superconducting Ring of $\mathrm{YBa}_{2} \mathrm{Cu}_{3} \mathrm{O}_{7-\delta}$, Phys. Rev. Lett. 73, 593 (1994).

[14] J. R. Kirtley, L. Paulius, A. J. Rosenberg, J. C. Palmstrom, C. M. Holland, E. M. Spanton, D. Schiessl, C. L. Jermain, J. Gibbons, Y.-K.-K. Fung, M. E. Huber, D. C. Ralph, M. B. Ketchen, G. W. Gibson, and K. A. Moler, Scanning SQUID susceptometers with submicron spatial resolution, Rev. Sci. Instrum. 87, 093702 (2016).

[15] J. R. Maze, P. L. Stanwix, J. S. Hodges, S. Hong, J. M. Taylor, P. Cappellaro, L. Jiang, M. V. Gurudev Dutt, E. Togan, A. S. Zibrov, A. Yacoby, R. L. Walsworth, and M. D. Lukin, Nanoscale magnetic sensing with an individual electronic spin in diamond, Nature 455, 644 (2008).

[16] G. Balasubramanian, I. Y. Chan, R. Kolesov, M. AlHmoud, J. Tisler, C. Shin, C. Kim, A. Wojcik, P. R. Hemmer, A. Krueger, T. Hanke, A. Leitenstorfer, R. Bratschitsch, F. Jelezko, and J. Wrachtrup, Nanoscale imaging magnetometry with diamond spins under ambient conditions, Nature 455, 644 (2008).

[17] P. Maletinsky, S. Hong, M. S. Grinolds, B. Hausmann, M. D. Lukin, R. L. Walsworth, M. Loncar, and A. Yacoby, A robust scanning diamond sensor for nanoscale imaging with single nitrogen-vacancy centres, Nat. Nanotech. 7, 320 (2012).

[18] L. Rondin, J.-P. Tetienne, P. Spinicelli, C. Dal Savio, K. Karrai, G. Dantelle, A. Thiaville, S. Rohart, J.-F. Roch, and V. Jacques, Magnetometry with nitrogen-vacancy defects in diamond, Appl. Phys. Lett. 100, 153118 (2012).

[19] H. F. Hess, R. B. Robinson, R. C. Dynes, J. M. Valles Jr., and J.V. Waszczak, Scanning-Tunneling-Microscope Observation of the Abrikosov Flux Lattice and the Density of States near and inside a Fluxoid, Phys. Rev. Lett. 62, 214 (1989).

[20] A. A. Abrikosov, On the magnetic properties of superconductors of the second group, Soviet Phys. JETP 5, 1174 (1957).

[21] M. Tinkham, Introduction to Superconductivity (McGrawHill, Inc., New-York, 1996), 2nd ed.

[22] A. Petrovic, PhD thesis, University of Geneva, 2009.

[23] Scanning tunneling spectroscopy of high-temperature superconductors, Rev. Mod. Phys. 79, 353 (2007). 